\title{
Quantifying scattering from dense media using two-dimensional impedance maps
}

\author{
Kazuki Tamura, ${ }^{1, a)}$ Jonathan Mamou, ${ }^{2}$ Kenji Yoshida, ${ }^{3}$ Tadashi Yamaguchi, ${ }^{3}$ and Emilie Franceschini ${ }^{4, b)}$ \\ ${ }^{1}$ Department of Innovative Medical Photonics, Preeminent Medical Photonics Education and Research Center, Hamamatsu University \\ School of Medicine, Hamamatsu, Shizuoka, Japan \\ ${ }^{2}$ Frederic L. Lizzi Center for Biomedical Engineering, Riverside Research, New York, New York, 10038, USA \\ ${ }^{3}$ Center for Frontier Medical Engineering, Chiba University, Chiba, Chiba, Japan \\ ${ }^{4}$ Aix-Marseille Université, CNRS, Centrale Marseille, LMA, Marseille, France
}

\begin{abstract}
:
A better understanding of ultrasound scattering in a three-dimensional (3D) medium can provide more accurate methods for ultrasound tissue characterization. The possibility of using two-dimensional impedance maps (2DZMs) based on correlation coefficients has shown promise in the case of isotropic and sparse medium [Luchies and Oelze, J. Acoust. Soc. Am. 139, 1557-1564 (2016)]. The present study investigates the use of 2DZMs in order to quantify 3D scatterer properties of dense media from two-dimensional (2D) histological slices. Two 2DZM approaches were studied: one based on the correlation coefficient and the other based on the 2D Fourier transform of 2DZMs. Both 2DZM approaches consist in estimating the backscatter coefficient (BSC) from several 2DZMs, and then the resulting BSC was fit to the theoretical polydisperse structure factor model to yield 3D scatterer properties. Simulation studies were performed to evaluate the ability of both 2DZM approaches to quantify scattering of a 3D medium containing randomly distributed polydisperse spheres or monodisperse ellipsoids. Experimental studies were also performed using the histology photomicrographs obtained from HT29 cell pellet phantoms. Results demonstrate that the 2DZM Fourier transform-based approach was more suitable than the correlation coefficient-based approach for estimating scatterer properties when using a small number of 2DZMs. (C) 2020 Acoustical Society of America. https://doi.org/10.1121/10.0001972
\end{abstract}

(Received 26 March 2020; revised 27 August 2020; accepted 30 August 2020; published online 23 September 2020)

[Editor: Keith A. Wear]

Pages: 1681-1691

\section{INTRODUCTION}

Quantitative ultrasound (QUS) techniques based on the backscatter coefficient (BSC) are well established in the field of ultrasound tissue characterization. The model-based approach consists in fitting a theoretical scattering model to the experimentally estimated BSC, and the fit parameters (called QUS estimates) can be directly related to tissue microstructure (e.g., scatterer size, shape, spatial organization, and scattering strength). The most popular scattering models are the spherical Gaussian model (SGM) ${ }^{1,2}$ and the fluid-filled sphere model (FFSM), ${ }^{3}$ which assume sparse media. The SGM, which was developed by Lizzi et al., 1,2 assumes that ultrasound scatterers are isotropic, sparse, and spherical with a continuous impedance fluctuation following a spherical Gaussian curve. The FFSM assumes that the ultrasound scatterers are sparse discrete fluid-filled spheres. ${ }^{3,4}$ QUS estimates have been successfully used to assess tumors in the eye, ${ }^{5}$ prostate, ${ }^{6}$ breast, ${ }^{7}$ lymph nodes, ${ }^{8}$ fatty liver, ${ }^{9}$ and thyroid. ${ }^{10}$ Another class of theoretical scattering models is based on a structure factor model (SFM), which can characterize sparse and concentrated media. ${ }^{11,12}$

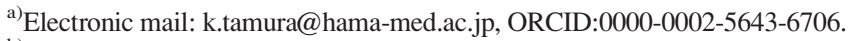

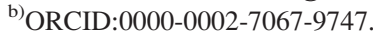

The SFM describes tissue as an ensemble of discrete scatterers and considers potential interference effects caused by coherent scattering using a structure factor. ${ }^{13,14}$ The SFM has proven to be the most appropriate model for modeling densely packed cells in in vitro cell pellet biophantoms ${ }^{15,16}$ and in ex vivo homogeneous mouse tumor models. ${ }^{17}$ However, even with advanced models, such as SFM, it remains challenging to establish a relationship between QUS scatterer property estimates and actual tissue microstructures (generally identified from optical microscopy images) in heterogeneous tissues. ${ }^{17,18}$

In many soft tissues, the tissue microstructure responsible for scattering remain unknown. That is why a method based on computational acoustic models, namely, threedimensional impedance maps (3DZMs), was developed as a means to predict ultrasonic scattering from complex tissue microstructures. ${ }^{19,20}$ In these previous studies, 3DZMs were derived from a three-dimensional (3D) histological volume set using signal and image processing methods. Specifically, the $3 \mathrm{D}$ histological volume set is created from a series of adjacent tissue sections that have been aligned to one another and appropriately stained to highlight specific tissue features. These tissue features are assigned acoustic impedance values to form a 3DZM. By assuming weak scattering, the $\mathrm{BSC}$ resulting from a plane wave incident on the tissue 
modeled by a 3DZM volume can be obtained by computing the squared magnitude of the Fourier transform of the 3DZM. Finally, a scattering model can be applied to extract QUS estimates. The QUS parameters estimated from 3DZMs are useful to help interpret QUS parameters obtained experimentally with ultrasound such as in the 3DZM study performed by Mamou et al. ${ }^{19}$ on complex mammary tumors. The effective scatterer radius estimated from 3DZM analysis can help suggest the anatomic structures responsible for scattering such as the acini in a fibroadenoma tumor ${ }^{19,20}$ or the hematocyte nuclei in the liver. ${ }^{21}$

However, obtaining 3DZMs using serial histology sectioning is time consuming and expensive because numerous sections must be made, affixed on a slide, stained, and optically digitized. Therefore, Luchies et al. ${ }^{22}$ proposed a theoretical method for estimating the effective scatterer radius using two-dimensional impedance maps (2DZMs) instead of 3DZMs. This method consists in obtaining the BSC from the 3D scattering volume by computing the Fourier transform of the spatial autocorrelation function of a 2DZM, which is equal to the magnitude squared of the Fourier transform of the 3DZM when considering isotropic and random media. This 2DZM correlation coefficient-based approach has proven to be as accurate as the classical 3DZM when considering simulations of sparse collections of scatterers and rabbit liver histological slices. ${ }^{22}$ More recently, numerical simulation on monodisperse spheres with a volume fraction of 30\% demonstrated that the 2DZM approach can capture information about the 3D spatial positioning of scatterers. ${ }^{23}$

The present study investigates the use of 2DZMs in order to quantify 3D scatterer properties of dense media from two-dimensional (2D) histological slices. Two 2DZM approaches were studied: one based on the correlation coefficient as proposed by Luchies et al., ${ }^{22}$ and the other based on the 2D Fourier transform of 2DZMs. Both 2DZM approaches consist in estimating the BSC from several 2DZMs, and then the resulting BSC was fit to the theoretical polydisperse SFM to yield 3D scatterer properties. Simulations of 2DZMs obtained from concentrated collections of scatterers (randomly distributed polydisperse spheres or monodisperse ellipsoids) are performed to evaluate the ability of 2DZM approaches to quantify scattering from 3D dense media. Then scatterer properties (such as scatterer size or volume fraction) estimated from both 2DZM approaches are compared. Finally, 2DZM approaches are compared on 2DZMs created from histology photomicrographs obtained from colon adenocarcinoma (HT29) cell pellets. This study contributes to the validation of 2DZM approaches in the case of dense media as a valuable alternative to 3DZM.

\section{BACKSCATTERING THEORY IN WEAK- SCATTERING MEDIA AND IMPEDANCE MAPS}

Assuming plane wave propagation, weak-scattering, far-field approximation, and statistically homogeneous media, the BSC in 3D space and the backscattering configuration is given by [see Eq. (58) in Ref. 24]

$$
\begin{aligned}
\operatorname{BSC}(k)= & \frac{k^{4}}{4 \pi^{2} V}\left(\left|\int\left\langle\gamma_{z}(\mathbf{r})\right\rangle e^{-i 2 \mathbf{k} \cdot \mathbf{r}} d^{3} \mathbf{r}\right|^{2}\right. \\
& +\left\langle\iint\left\langle\left(\gamma_{z 1}-\left\langle\gamma_{z 1}\right\rangle\right)\left(\gamma_{z 2}-\left\langle\gamma_{z 2}\right\rangle\right)\right\rangle\right. \\
& \left.\left.\times e^{-i 2 \mathbf{k} \cdot\left(\mathbf{r}_{1}-\mathbf{r}_{2}\right)} d^{3} \mathbf{r}_{1} d^{3} \mathbf{r}_{2}\right\rangle\right),
\end{aligned}
$$

where (2k) is the backscattering vector, $k$ is the wavenumber, $V$ is the volume containing the scattering source, $\gamma_{z}$ is the relative contrast in acoustic impedance at position $\mathbf{r}$. For convenience, we have written $\gamma_{z 1}=\gamma_{z}\left(\mathbf{r}_{1}\right)$ and $\gamma_{z 2}=\gamma_{z}\left(\mathbf{r}_{2}\right)$, where $\mathbf{r}_{1}$ and $\mathbf{r}_{2}$ represent two positions in the medium. The symbol \langle\rangle represents the ensemble average. In Eq. (1), the first term is the coherent scattering contribution, and the second term is generally identified as the incoherent scattering contribution when considering independently and uniformly distributed scatterers. However, this second term cannot be labeled "incoherent" when considering dense media because, in this case, the autocovariance function $\left\langle\left(\gamma_{z 1}-\left\langle\gamma_{z 1}\right\rangle\right)\left(\gamma_{z 2}-\left\langle\gamma_{z 2}\right\rangle\right)\right\rangle$ would contain contributions from correlations among scatterer positions.

By neglecting the coherent scattering contribution in the first term of Eq. (1) and assuming isotropic medium, the BSC in 3D space reduces to ${ }^{24}$

$$
\operatorname{BSC}_{\mathrm{CC}}(k)=\frac{k^{3}}{2 \pi}\left\langle\left|\gamma_{z}-\left\langle\gamma_{z}\right\rangle\right|^{2}\right\rangle \int_{0}^{\infty} b_{\gamma_{z}}(\Delta r) \sin (2 k \Delta r) \Delta r d \Delta r,
$$

where $b_{\gamma_{z}}$ is the correlation coefficient of $\gamma_{z}$, and $\Delta r$ is the lag distance, i.e., the length of the vector $\left(\mathbf{r}_{1}-\mathbf{r}_{2}\right) .{ }^{25}$ The correlation coefficient depends on the microstructure of the scattering medium and verifies $b_{\gamma_{z}}(0)=1$ and $b_{\gamma_{z}}(\infty)=0$.

Equations (1) and (2) introduced the theoretical background needed to understand the relationship between ultrasonic backscattering and 2DZM. When considering isotropic media, the BSC in 3D space can be estimated from the correlation coefficient $b_{\gamma_{z}}$, which can be calculated from the 2DZMs.

\section{2D IMPEDANCE COMPUTATIONAL MODELS}

In this section, we recall briefly the 2DZM approach based on the correlation coefficient, ${ }^{22}$ and then we present the 2DZM Fourier transform-based approach. Both 2DZM approaches consist in estimating the BSC from several 2DZMs, and then the theoretical polydisperse SFM is fit to the estimated BSC. The fit parameters can provide a meaningful description of the tissue microstructure. Note that without loss of generality, in the remainder of the paper, the term "2DZM" (or "3DZM") is used to represent the 2D impedance contrast map (or the 3D impedance contrast map, respectively), i.e., the map of $\gamma_{z}$ rather than that of $z$.

\section{A. 2DZM correlation coefficient-based approach}

The 2DZM correlation coefficient-based approach consists in computing the correlation coefficient $b_{\gamma_{2}}$ from 
2DZMs to evaluate the $\mathrm{BSC}_{\mathrm{CC}}$ in $3 \mathrm{D}$ space. More specifically, for each 2DZM, the mean value of $\gamma_{z}$ is subtracted from the impedance contrast map in order to obtain $\gamma_{z}^{\prime}=\gamma_{z}$ $-\left\langle\gamma_{z}\right\rangle$. The autocovariance function $C_{\gamma_{z}}$ was then computed as

$$
C_{\gamma_{z}}\left(\mathbf{r}_{g, h}\right)=\sum_{j, l=0}^{N} \gamma_{z}^{\prime}\left(\mathbf{r}_{g, h}+\mathbf{r}_{j, l}^{\prime}\right) \gamma_{z}^{\prime}\left(\mathbf{r}_{j, l}^{\prime}\right)
$$

where $j$ denotes the $x$ axis index, $l$ denotes the $y$ axis index, and $\mathbf{r}_{g, h}$ denotes the lag position for the $g$ th and $h$ th indices. Radial lines from $C_{\gamma_{z}}\left(\mathbf{r}_{g, h}\right)$ are then averaged to obtain $C_{\gamma_{z}}(\Delta r)$. The correlation coefficient was obtained as $b_{\gamma_{z}}(\Delta r)$ $=C_{\gamma_{z}}(\Delta r) /\left|\gamma_{z}-\left\langle\gamma_{z}\right\rangle\right|^{2}$. In practice, the estimation of $b_{\gamma_{z}}(\Delta r)$ can be biased for large lag distances if a small number of 2DZMs is used. (This point will be discussed further in Sec. VI A.) Therefore, the correlation coefficient $b_{\gamma_{z}}(\Delta r)$ was set to zero outside the lag distance $0 \leq \Delta r \leq 6 \hat{a}$. The scatterer radius $\hat{a}$ corresponds to the radius estimated by fitting the fluid-filled sphere correlation coefficient model to the estimated correlation coefficient. The fluid-filled sphere correlation coefficient model is expressed as ${ }^{24}$

$$
b_{\gamma_{z}}^{\text {theo }}(\Delta r)= \begin{cases}1-\frac{3 \Delta r}{4 \hat{a}}+\frac{(\Delta r)^{3}}{16 \hat{a}^{3}}, & \text { for } 0 \leq \Delta r \leq 2 \hat{a}, \\ 0, & \text { for } \Delta r \geq 2 \hat{a} .\end{cases}
$$

For a collection of several 2DZMs, $b_{\gamma_{z}}(\Delta r)$ was estimated for each 2DZM, and the resulting averaged $b_{\gamma_{z}}(\Delta r)$ allows us to estimate the $\mathrm{BSC}_{\mathrm{CC}}$ as given by Eq. (2). Finally, the resulting $\mathrm{BSC}_{\mathrm{CC}}$ was fitted to the theoretical polydisperse SFM in 3D space in order to yield QUS parameters. [The theoretical BSC using the polydisperse SFM in 3D space is given by Eq. (4) in Ref. 16.]

\section{B. 2DZM Fourier transform-based approach}

The 2DZM Fourier transform-based approach focuses on $2 \mathrm{D}$ space and estimated the $\mathrm{BSC}_{2 \mathrm{DFT}}$ from $2 \mathrm{DZM}$ slices. The $3 \mathrm{D}$ scatterer properties (such as the effective scatterer size) are then estimated by fitting the estimated BSC $_{2 D F T}$ with a scattering model in 2D space.

The BSC in 2D space is calculated by computing 2D Fourier transforms of several 2DZMs and averaging the radial (frequency) lines as

$$
\mathrm{BSC}_{2 \mathrm{DFT}}(k)=\frac{k^{3}}{4 \pi^{2}} E\left[|\mathrm{FT}(2 \mathrm{DZM})|^{2}\right] \frac{1}{L^{2}}\left(\frac{L}{N_{p}}\right)^{4},
$$

where $E$ denotes the expected values, $L^{2}$ is the surface of a $2 \mathrm{D}$ region-of-interest (ROI) divided into $N_{p}^{2}$ pixels to obtain the 2DZMs, and FT(2DZM) represents a radial line of the 2D Fourier transform of a 2DZM matrix (with the line being in the direction of the incident wave). The dependence in $k^{3}$ comes from the scattering in $2 \mathrm{D}$ space as shown in Eq. (A2) in the Appendix. The term $\left(1 / L^{2}\right)$ allows us to compute the backscatter by unit area. The 2D Fourier transforms give the $\mathrm{BSC}_{2 \mathrm{DFT}}$ values on a centered grid of wavevectors between $\pm \pi N_{p} /(2 L)$ with a step of $\pi / L$. The theoretical polydisperse $S F M$ in $2 \mathrm{D}$ space is then fit to the estimated $\mathrm{BSC}_{2 \mathrm{DFT}}$ to yield QUS parameters. The polydisperse SFM in 2D space considers an ensemble of discs differing only in size and is detailed in the Appendix.

In this 2DZM approach, it is assumed that a given 3DZM contains a single type of scatterer/sphere differing only in size, and the distribution of the sphere radii $\tau$ follows a gamma probability density function (PDF) given by

$$
f_{(\xi, \bar{a})}(\tau)=\frac{1}{\xi !}\left(\frac{\xi+1}{\bar{a}}\right)^{\xi+1} \tau^{\xi} e^{-(\xi+1) \tau / \bar{a}},
$$

where $\bar{a}$ is the mean radius and $\xi$ is the gamma width factor, $\xi>-1$, which measures the width of the sphere radius distribution. The 2DZMs obtained from perfect cross sections of 3DZM contain an ensemble of polydisperse discs. The disc radius PDF $p(r)$ of the planar section of the 3DZM can be obtained from the convolution between the sphere radius $\operatorname{PDF} f_{(\xi, \bar{a})}$ and the distribution of radii inside a sphere of radius $\tau$ as $^{26}$

$$
p(r)=2 r \int_{r}^{\infty} \frac{f_{(\xi, \bar{a})}(\tau)}{\sqrt{\left(\tau^{2}-r^{2}\right)}} d \tau .
$$

\section{MATERIAL AND METHODS}

\section{A. Simulations}

Simulation studies were performed to evaluate the ability of 2DZM approaches to estimate the scatterer properties of a simulated 3D medium (i.e., scatterer size, surface, or volume fraction). Scatterers were uniformly randomly distributed with non-overlapping positions using a Monte Carlo algorithm (see Sec. II B A in Ref. 27). The simulated volume was fixed to $L^{3}=280 \times 280 \times 280 \mu \mathrm{m}^{3}$.

First, 3D media containing polydisperse spheres were simulated to study the performance of 2DZM approaches. The mean radius $\bar{a}$ and the standard deviation of the radius distribution $\sigma$ were chosen to be equal to $\bar{a}=4.5 \mu \mathrm{m}$ and $\sigma=0.66 \mu \mathrm{m}(\xi=45)$. Second, collections of randomly oriented monodisperse prolate ellipsoids were simulated with axial ratio 1.5 (major axis of $5.89 \mu \mathrm{m}$ and minor axis of $3.93 \mu \mathrm{m}$ ), and prolate ellipsoids of axial ratio 2 (major axis of $7.14 \mu \mathrm{m}$ and minor axis of $3.57 \mu \mathrm{m}$ ). Note that the volumes of both types of prolate ellipsoids were equal to the volume of a sphere of radius $4.5 \mu \mathrm{m}$. For the two sets of simulations, volume fractions ranging from $0.6 \%$ to $30 \%$ were investigated.

The simulated volume was sampled in $N_{p}^{3}=512 \times 512$ $\times 512$ voxels and the $3 \mathrm{DZM}$ was constructed by arbitrarily considering a relative impedance contrast equal to $\gamma_{z}=0.11$ within scatterers and $\gamma_{z}=0$ elsewhere. Then, a collection of 2DZMs was obtained from the 3DZM by performing perfect 
cross sections in the $X Y$ plane at regular spacing (every 18 $\mu \mathrm{m})$ in the $Z$ axis as shown in Fig. 1 .

Note that the surface fractions of the discs obtained from the 2DZMs were approximately equal to the volume fractions when perfect cross sections were used to generated 2DZMs: the averaged surface fractions were equal to $0.8 \%$, $2.8 \%, 5.7 \%, 12.2 \%, 17.7 \%, 23.6 \%$, and $29.3 \%$ for the studied volume fractions of $0.6 \%, 3 \%, 6 \%, 12 \%, 18 \%, 24 \%$, and $30 \%$, respectively. Therefore, in the remainder of the paper, the theoretical surface fraction values will be approximated by the prescribed volume fraction values.

\section{B. Experiments}

The 2DZM approaches were also tested using digital histology photomicrographs from thin sections obtained from HT29 cell pellet biophantoms. Cell pellet biophantoms consist of centrifuged cells mimicking densely packed cells in tumors and serve as simplified in vitro versions of real tumors. The detailed procedure for constructing cell pellet biophantoms is as follows. HT29 cells were grown at $37^{\circ} \mathrm{C}$ in T175 flasks with Dulbecco's modified Eagles's medium containing 4.5 grams of glucose per liter and supplemented with $10 \%$ fetal calf serum. For forming one cell pellet, around $2 \times 10^{8}$ HT29 cells are needed. Cells from the T175 flasks were detached with accutase and washed in phosphate-buffered saline (PBS). Cells from flasks were collected and after homogenization with a pipette tip, $50 \mu \mathrm{L}$ of this cell suspension was withdrawn for cell size analysis. The remaining suspension was centrifuged for $5 \mathrm{~min}$ at $1200 \mathrm{~g}$, and then the medium was aspirated and $500 \mu \mathrm{L}$ of PBS $+/+$ was added. After homogenization with a pipette tip, the cells were transferred in an eight-well Nunc Lab-Tek II Chamber Slide System (Dominique Dutscher, Brumath, France) and finally centrifuged for $5 \mathrm{~min}$ at $1700 \mathrm{~g}$ to form densely packed cell pellets. Finally, the cell samples were fixed in 10\% buffered formalin for three days, dehydrated in graded ethanol series, cleared in methylcyclohexane, and embedded in methyl methacrylate resin before being sectioned and stained with toluidine blue. The procedure was repeated two times to form two cell pellet biophantoms.

To create the 2DZM, two impedance values were used: ${ }^{28} 1.84$ MRayl for the nuclei and 1.66 MRayl for the

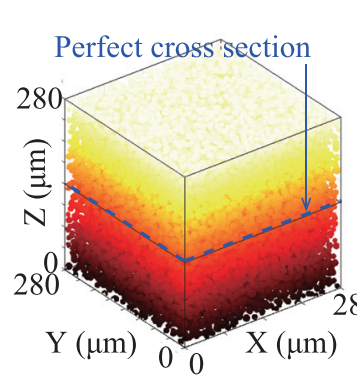

(a)

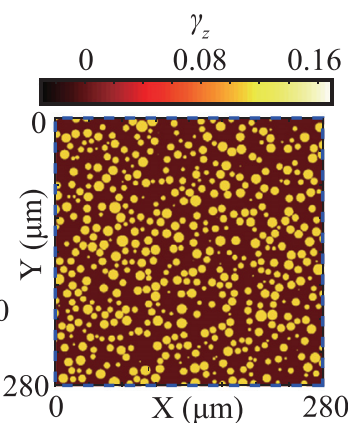

(b)
FIG. 1. (Color online) (a) Example of a 3DZM. (b) Corresponding 2DZM obtained from a perfect cross section in the $X Y$ plane of the 3DZM. cytoplasm and extracellular matrix. The nuclei were obtained by manual segmentation. Therefore, the nuclei are considered to be the main source of scattering in the proposed 2DZM computational study. The resolution of the resulting 2DZM was $0.21 \mu \mathrm{m} /$ pixel. Six non-overlapping ROIs of sizes $512 \times 512$ pixels $\left(L^{2} \approx 107.5 \times 107.5 \mu \mathrm{m}\right)$ were selected at the center of the $2 \mathrm{DZM}$ in order to compute the $\mathrm{BSC}_{\mathrm{CC}}$ when using the $2 \mathrm{DZM}$ correlation coefficientbased approach, or compute $\mathrm{BSC}_{2 \mathrm{DFT}}$ when using the 2DZM Fourier transform-based approach. Herein, the ROI pixel size and spatial resolution is determined by the magnification used to digitize the histology photomicrograph (i.e., here $20 \times$ ). The representative ROI is shown in Fig. 2 .

The actual radius, gamma width factor, and surface fraction of the nuclei were also computed from manually segmenting the histology photomicrographs. Segmented nuclei were approximated by ellipses in order to deduce the semi-minor axis $b^{\prime}$ and axial ratio $\nu^{\prime}$ of the fitted ellipses and compute the actual effective scatterer radius $a^{\prime}=b^{\prime} \sqrt{\nu^{\prime}}$. The surface fraction was the manually segmented area divided by the total image area. The effective scatterer radius of the segmented nuclei was found to be equal to $4.6 \mu \mathrm{m}$ for cell pellet 1 and $4.5 \mu \mathrm{m}$ for cell pellet 2 . The gamma width factor $\xi$ was obtained by fitting the distribution of the nuclear disc radii with $p(r)$ given by Eq. (7), and was found equal to 66 for cell pellet 1 and 164 for cell pellet 2 . The surface fractions were found equal to $25 \%$ for both cell pellets.

\section{QUS parameter estimation}

The $\mathrm{BSC}_{\mathrm{CC}}$ and $\mathrm{BSC}_{2 \mathrm{DFT}}$ are computed from a collection of at least six independent cross sections. When using the 2DZM correlation coefficient-based approach, the $\mathrm{BSC}_{\mathrm{CC}}$ was estimated in $3 \mathrm{D}$ space, and the QUS parameters were obtained by fitting the $\mathrm{BSC}_{\mathrm{CC}}$ with the polydisperse SFM in 3D space. When using the 2DZM Fourier transform-based approach, the $\mathrm{BSC}_{2 \mathrm{DFT}}$ was estimated in $2 \mathrm{D}$ space, and the QUS parameters were obtained by fitting the $\mathrm{BSC}_{2 \mathrm{DFT}}$ with the polydisperse SFM in 2D space. For both 2DZM approaches, the fitting bandwidth was set to $20-200$ MHz. The lowest frequency of $20 \mathrm{MHz}$ was chosen because the $\mathrm{BSC}_{2 \mathrm{DFT}}$ curves are unreliable for frequencies lower than $c / L \approx 15 \mathrm{MHz}$ (where $c$ is the speed of sound assumed to be equal to $1500 \mathrm{~m} / \mathrm{s}$ ) in the case of the histology photomicrograph sample. The fitting procedure simultaneously yielded four QUS parameters: the mean scatterer sphere
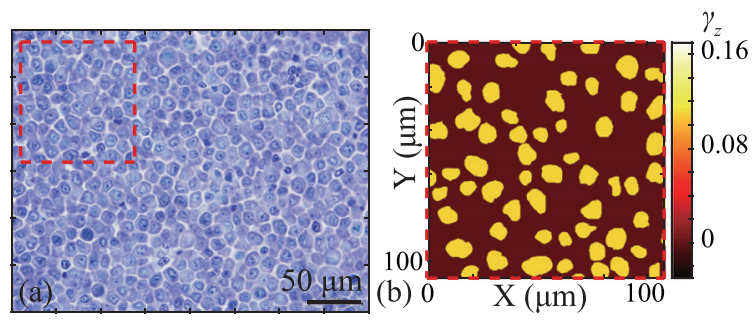

FIG. 2. (Color online) (a) Typical example of the histology photomicrograph from the cell pellet biophantom. (b) Corresponding 2DZM obtained from the histology photomicrograph within the red dashed square. 
radius $a^{*}$, the standard deviation of the radius distribution $\sigma^{*}=a^{*} / \sqrt{\xi^{*}+1}$, the impedance contrast $\gamma_{z}^{*}$ and the total surface fraction in 2D space $\phi^{*}$ (or the total volume fraction in 3D space).

The relative errors for the estimated QUS parameters, denoted $P^{*}$, are given by $\left(P^{*}-P_{\text {true }}\right) / P_{\text {true }}$, where $P_{\text {true }}$ is the actual value of $P$ (i.e., actual sphere size $a$, standard deviation of radius distribution $\sigma$, surface or volume fraction $\phi$, or impedance contrast $\gamma_{z}$ ). For the simulated media, each reported QUS value corresponds to the estimated QUS parameters average obtained from five independent realizations of 2DZMs with the same numerical phantom parameters.

\section{RESULTS}

\section{A. Comparison between simulated $\mathrm{BSC}_{3 \mathrm{DFT}}$ and $\mathrm{BSC}_{\mathrm{CC}}$}

Figure 3(a) gives the correlation coefficient $b_{\gamma}$ for the simulated 3D media, consisting of polydisperse spheres at volume fractions of $0.6 \%, 12 \%$, and $30 \%$. The $b_{\gamma}$ are given in solid lines when using 6 2DZM slices (blue lines) or 120 2DZM slices (red lines). The dashed lines in Fig. 3(a) represent the fluid-filled sphere correlation coefficient model $b_{\gamma}^{\text {theo }}$, given by Eq. (4). As expected, the estimated $b_{\gamma}$ deviates from the fluidfilled sphere correlation coefficient model as the volume fraction increases because this model is only valid for sparse media. The corresponding $\mathrm{BSC}_{\mathrm{CC}} / k^{4}$ computed with the 2DZM correlation coefficient-based approach are given in solid lines in Fig. 3(b). Also given in Fig. 3(b) are the $\mathrm{BSC}_{3 \mathrm{DFT}}$ obtained from the 3D Fourier transform of 3DZMs and computed as follows: $\operatorname{BSC}_{3 \mathrm{DFT}}(k)=C k^{4} E\left[|\mathrm{FT}(3 \mathrm{DZM})|^{2}\right]$, where
$C$ is a proportionality constant. The $\mathrm{BSC}_{3 \mathrm{DFT}}$ is considered as the exact computation of the BSC in $3 \mathrm{D}$ space. Good agreement is obtained between the $\mathrm{BSC}_{3 \mathrm{DFT}}$ and $\mathrm{BSC}_{\mathrm{CC}}$ when using 120 2DZM slices. However, large differences (up to $20 \mathrm{~dB}$ ) are observed between the $\mathrm{BSC}_{3 \mathrm{DFT}}$ and $\mathrm{BSC}_{\mathrm{CC}}$ when using only six 2DZM slices for the lower volume fractions of $0.6 \%$ and $12 \%$, especially at frequencies larger than $90 \mathrm{MHz}$. These large differences are discussed in Sec. VIA.

\section{B. Comparison between simulated $\mathrm{BSC}_{3 \mathrm{DFT}}$ and $\mathrm{BSC}_{2 \mathrm{DFT}}$}

The simulated $\mathrm{BSC}_{3 \mathrm{DFT}}$ and $\mathrm{BSC}_{2 \mathrm{DFT}}$ obtained from the same $3 \mathrm{D}$ polydisperse sphere distribution are compared in order to assess how the 2D BSC derived from the 2DZM Fourier transform-based approach can be linked to the actual 3D backscattering. Examples of 3D sphere radius histograms and corresponding 2D disc radius histograms obtained from six 2DZMs are shown in Fig. 4(a). The corresponding 3D BSC $\left[\mathrm{BSC}_{3 \mathrm{DFT}} / k^{4}\right]$ and 2D BSC $\left[\mathrm{BSC}_{2 \mathrm{DFT}} / k^{3}\right.$ $\times(4 a / 3)]$ are compared in Fig. 4(b). The normalization of $\mathrm{BSC}$ by $k^{4}$ in $3 \mathrm{D}$ and $k^{3}$ in 2D removes the frequency dependence of Rayleigh scattering. The $\mathrm{BSC}_{2 \mathrm{DFT}}$ is multiplied by $4 a / 3=V_{s} / A_{s}$ in order to compare the BSC amplitudes in $2 \mathrm{D}$ and $3 \mathrm{D}$ spaces because the backscattering is proportional to the scatterer volume $V_{s}$ in the 3D space (spheres) and the scatterer surface $A_{s}$ in the 2D space (discs) in the Rayleigh scattering regime.

Overall, $\left[\mathrm{BSC}_{3 \mathrm{DFT}} / k^{4}\right]$ and $\left[\mathrm{BSC}_{2 \mathrm{DFT}} / k^{3} \times(4 a / 3)\right]$ have similar amplitudes as a function of volume fractions. Moreover, $\left[\mathrm{BSC}_{3 \mathrm{DFT}} / k^{4}\right]$ and $\left[\mathrm{BSC}_{2 \mathrm{DFT}} / k^{3} \times(4 a / 3)\right]$ curves have similar shapes with similar frequency positions
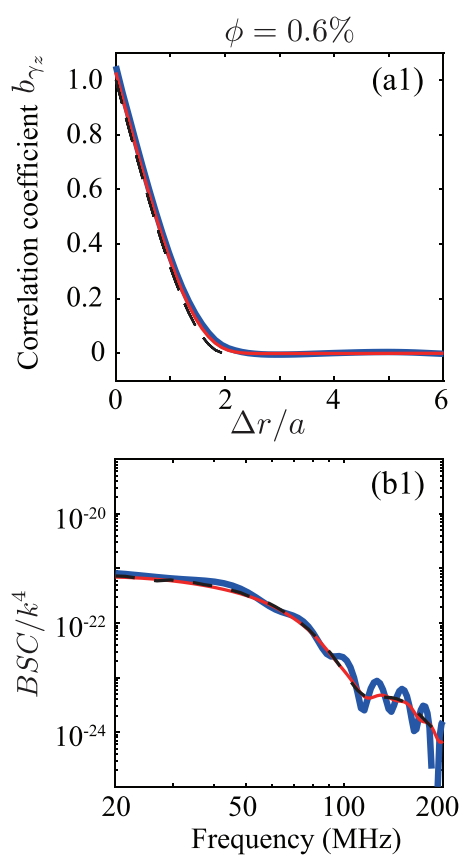
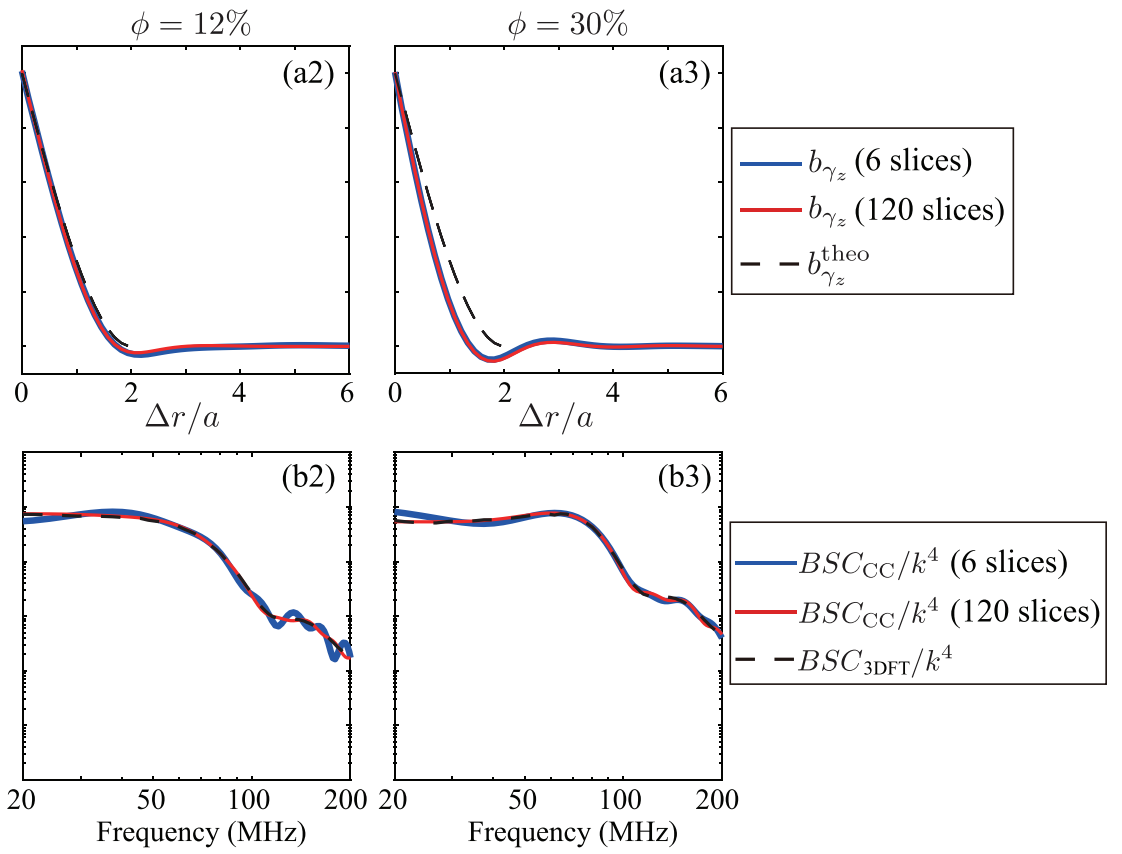

FIG. 3. (Color online) (a) Examples of correlation coefficients $b_{\gamma_{z}}$ for the simulated 3D media consisting of polydisperse spheres at volume fractions of $0.6 \%, 12 \%$, and $30 \%$. The $b_{\gamma_{z}}$ are estimated by using 6 2DZM slices (blue lines) or 120 2DZM slices (red lines). Also noted by dashed lines is the fluid-filled sphere correlation coefficient model $b_{\gamma}^{\text {theo }}$ given by Eq. (4). (b) Corresponding $\mathrm{BSC}_{\mathrm{CC}} / k^{4}$ computed from the $2 \mathrm{DZM}$ correlation coefficient-based approach by using $62 \mathrm{DZM}$ slices (blue lines) or $1202 \mathrm{DZM}$ slices (red lines). The $\mathrm{BSC}_{\mathrm{CC}}$ is computed by setting $b_{\gamma_{z}}$ ( $\Delta r$ ) to zero outside the lag distance $0 \leq \Delta r \leq 6$ $\hat{a}$. Also noted by dashed lines are the $\mathrm{BSC}_{3 \mathrm{DFT}} / k^{4}$ computed from 3D Fourier transforms of 3DZMs. 

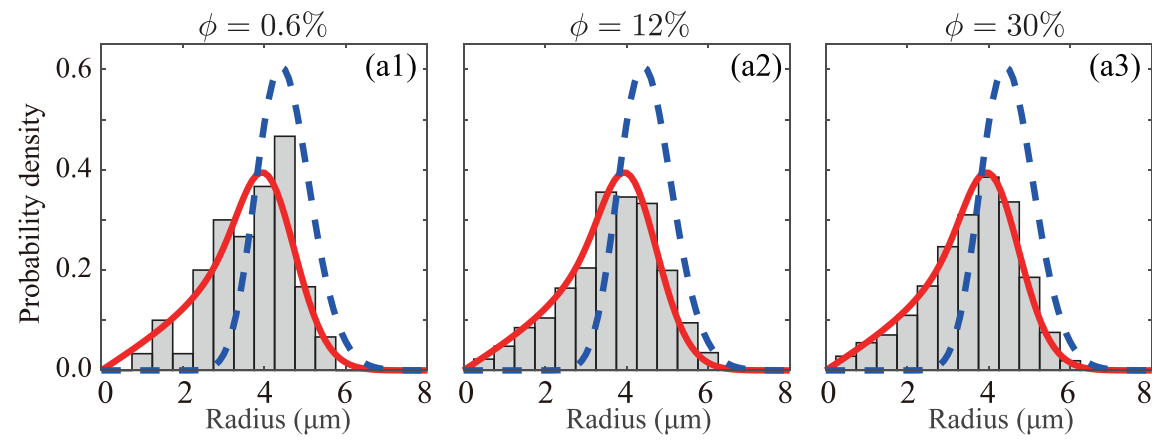

ए Observed radii

- $f_{(\xi, \bar{a})}(\tau)$

$p(r)$
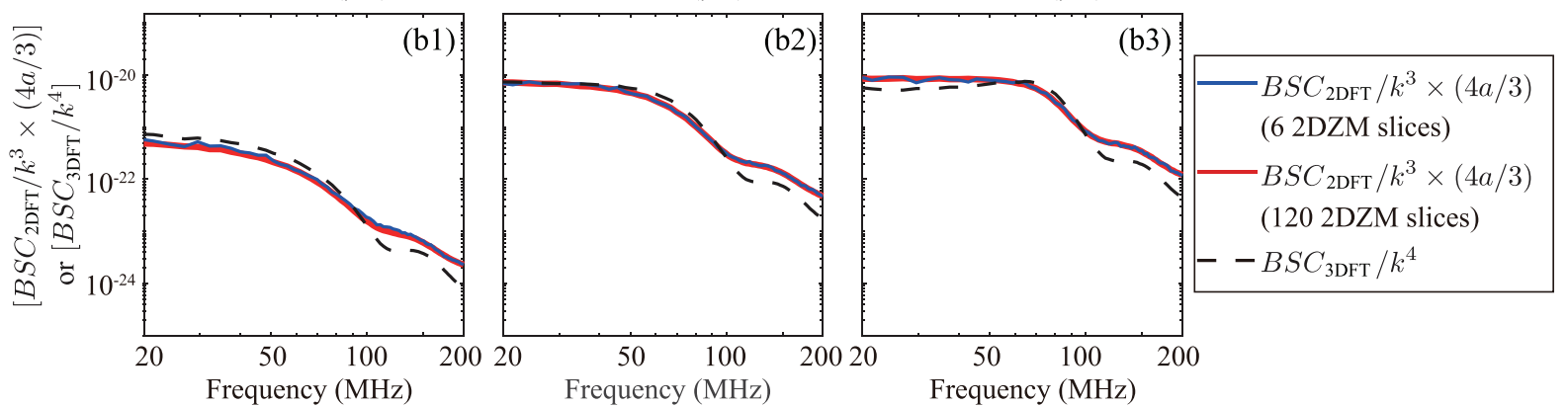

FIG. 4. (Color online) (a) Examples of a disc radius histogram obtained from six 2DZM slices for the surface fractions of $0.6 \%$, $12 \%$, and $30 \%$. The solid red line represents the disc radius probability density function (PDF) given by Eq. (7) and the dashed blue line represents the sphere radius PDF given by Eq. (6). (b) Examples of $\mathrm{BSC}_{2 \mathrm{DFT}} / k^{3} \times(4 a / 3)$ (solid lines) and $\mathrm{BSC}_{3 \mathrm{DFT}} / k^{4}$ computed from $3 \mathrm{D}$ Fourier transforms of $3 \mathrm{DZMs}$ (dashed lines). The $\mathrm{BSC}_{2 \mathrm{DFT}}$ are estimated by using 6 2DZM slices (blue lines) or 120 2DZM slices (red lines).

of BSC maxima. Indeed, the $\left[\mathrm{BSC}_{3 \mathrm{DFT}} / k^{4}\right]$ and $\left[\mathrm{BSC}_{2 \mathrm{DFT}} /\right.$ $\left.k^{3} \times(4 a / 3)\right]$ curves are flat in the Rayleigh scattering regime for frequencies less than $40 \mathrm{MHz}$, corresponding to $k a<0.75 \quad(a=4.5 \mu \mathrm{m})$. Then the $\left[\mathrm{BSC}_{3 \mathrm{DFT}} / k^{4}\right]$ and $\left[\mathrm{BSC}_{2 \mathrm{DFT}} / k^{3} \times(4 a / 3)\right]$ amplitudes decrease in the $50-90 \mathrm{MHz}$ frequency range, corresponding to $0.95 \leq k a$ $\leq 1.7$, for which the first maximum of BSC occurs. Finally, note that the $\mathrm{BSC}_{2 \mathrm{DFT}}$ computed by using 6 or $1202 \mathrm{DZM}$ slices are similar.

\section{QUS parameters estimated from 2DZM approaches}

Figures 5(a)-5(d) summarize QUS parameters $a^{*}, \sigma^{*}$, $\phi^{*}, \gamma_{z}^{*}$ estimated from both 2DZM approaches for the simulated 3D media, consisting of polydisperse spheres for volume fractions ranging from $0.6 \%$ to $30 \%$.

When considering the 2DZM correlation coefficientbased approach for estimating QUS parameters, the $\mathrm{BSC}_{\mathrm{CC}}$ is computed by using a small (6) or large (120) number of 2DZM slices. The QUS parameters estimated by using 120 independent 2DZM slices show a good agreement with actual parameters. The maximum relative errors of mean values are equal to $-2.2 \%, 3.0 \%,-16 \%, 18 \%$ for the parameters $a^{*}, \sigma^{*}, \phi^{*}, \gamma_{z}^{*}$, respectively. It is noticed that the standard deviations of $a^{*}$ and $\sigma^{*}$ are small, i.e., less than $1.2 \%$ and $5.8 \%$ of their mean values, respectively. Whereas the QUS parameters estimated by using six independent 2DZM slices show large fluctuations, the standard deviation of $a^{*}$ reaches values up to $20 \%$ of the mean value. The maximum relative errors of mean values are equal to $-8.2 \%,-36 \%,-70 \%$, $136 \%$ for the parameters $a^{*}, \sigma^{*}, \phi^{*}, \gamma_{z}^{*}$, respectively.

The 2DZM Fourier transform-based approach gives very similar QUS estimates when using 6 or 120 DZM slices so only the QUS estimates obtained from 6 2DZM slices are given in Fig. 5. One can observe a systematic bias of about $0.15 \mu \mathrm{m}$ for $a^{*}$ estimates (corresponding to relative errors of $3.4 \%$ ) and about approximately $2.7 \%$ for $\phi^{*}$ estimates (corresponding to relative errors less than $23 \%$ for
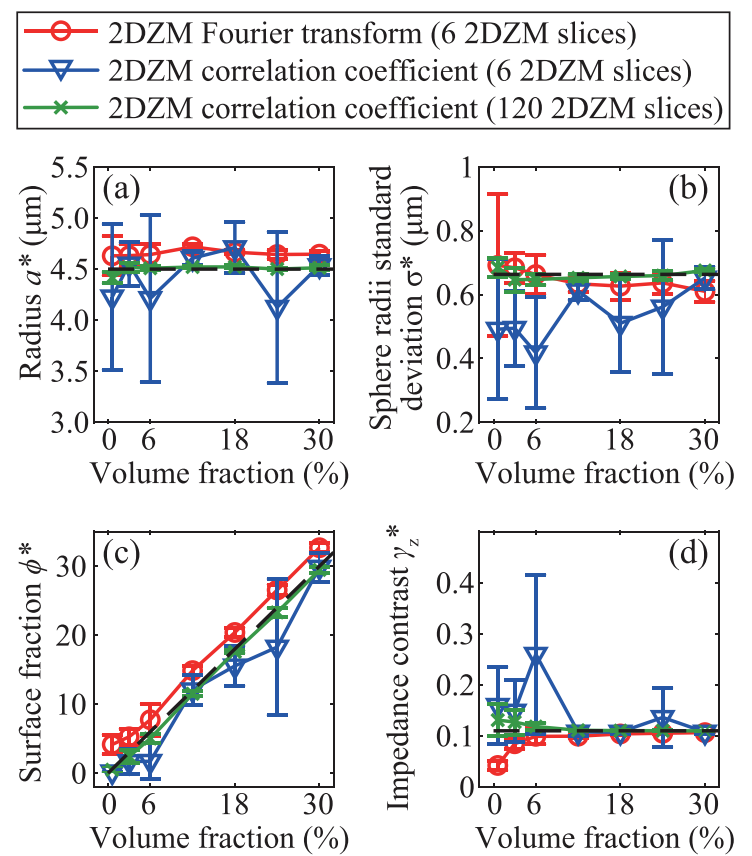

FIG. 5. (Color online) Parameters $\left(a^{*}, \sigma^{*}, \phi^{*}\right.$ and $\left.\gamma_{z}^{*}\right)$ as a function of actual volume fraction. The QUS parameters are estimated by the 2DZM correlation coefficient-based approach (when each $\mathrm{BSC}_{\mathrm{CC}}$ is computed with 6 or 120 2DZM slices) or the 2DZM Fourier transform-based approach (when each $\mathrm{BSC}_{2 \mathrm{DFT}}$ is computed with six 2DZM slices). The dashed black lines represent the actual parameters. 
$\phi \geq 12 \%$ ). The difference between the maximum and minimum values of radius $a^{*}$ is $0.2 \%$, which is smaller than the difference obtained with the 2DZM correlation coefficientbased approach by using the same number of 2DZM slices (i.e., six 2DZM slices).

Table I summarizes the QUS parameters $\left(a^{*}, \sigma^{*}, \phi^{*}\right.$, $\left.\gamma_{z}^{*}\right)$ estimated from both 2DZM approaches using six 2DZM slices for the cases of simulated ellipsoids and realistic spatial distributions of cells. Mean \pm standard deviation are computed from five independent realizations of 2DZMs (i.e., $5 \times 62 \mathrm{DZMs}$ ) for the simulated ellipsoids and three independent realizations of $2 \mathrm{DZMs}$ (i.e., $3 \times 62 \mathrm{DZMs}$ ) for the histological slices of HT29 cell pellets.

In the case of randomly oriented prolate ellipsoids (first two columns of Table I), both scatterer radius and surface fraction are satisfactorily estimated by the two 2DZM approaches with absolute relative errors less than $6.6 \%$ when the actual axial ratio $\nu=1.5$. However, for ellipsoids with the largest axial ratio $\nu=2.0$, both scatterer radius and surface fraction are underestimated with a relative error up to $-20 \%$.

In the case of realistic spatial distributions of cellular structures within cell pellets (last two columns of Table I), the scatterer radii $a^{*}$ and relative impedance difference $\gamma_{z}^{*}$ estimated by the 2DZM Fourier transform-based approach agree well with the expected values (with relative errors less than $5 \%$ and $9 \%$, respectively), whereas the surface fractions $\phi^{*}$ are overestimated. The scatterer radius $a^{*}$, estimated by the 2DZM correlation coefficient, varies between 3.4 and $5.9 \mu \mathrm{m}$ (corresponding to relative errors up to $31 \%$ ). Finally, one can notice that the QUS parameters estimated by the 2DZM correlation coefficient-based approach show larger standard deviations (i.e., 53 times larger for $\phi^{*}$ ) than those estimated by the 2DZM Fourier transform-based approach.

\section{DISCUSSION}

\section{A. Advantages and disadvantages of both 2DZM approaches}

The present study compares two different 2DZM approaches: one based on the correlation coefficient and the other based on the 2D Fourier transform of 2DZMs.
The advantage of the 2DZM correlation coefficient-based approach is the estimation of the $\mathrm{BSC}_{\mathrm{CC}}$ in $3 \mathrm{D}$ space. However, this approach requires a large number of 2DZMs because at least 120 slices was necessary to obtain a satisfactory match between $\mathrm{BSC}_{\mathrm{CC}}$ and $\mathrm{BSC}_{3 \mathrm{DFT}}$ [Fig. 3(b)]. This result is in line with the study of Luchies et al., ${ }^{23}$ showing that 2DZMs based on the correlation coefficient can be used to capture information about the $3 \mathrm{D}$ spatial positioning of scatterers when using a large number of 2DZM slices (12 800 2DZM slices in Ref. 23). It is noticed that the largest differences between $\mathrm{BSC}_{\mathrm{CC}}$ and $\mathrm{BSC}_{3 \mathrm{DFT}}$ are obtained when using only 6 2DZM slices for the lower volume fractions $\phi \leq 12 \%$ [Figs. 3(b1) and 3(b2)] because six independent 2DZM slices did not provide sufficient data for the ensemble average of $b_{\gamma_{z}}$ to converge (as illustrated in the supplemental figure, ${ }^{29}$ showing the $b_{\gamma_{z}}$ calculated using 1, 6, or 120 2DZM slices). The $b_{\gamma_{z}}$ curves obtained from a single 2DZM slice differ more from each other for lower volume fractions when compared to higher volume fractions, thus, the number of 2DZM slices should be increased for lower volume fractions. In the case of the lowest volume fraction of $0.6 \%$, the discrepancy between $\mathrm{BSC}_{3 \mathrm{DFT}}$ and $\mathrm{BSC}_{\mathrm{CC}}$ is due to the estimation of $b_{\gamma_{z}}(\Delta r \geq 2 a)$, which should be equal to zero but is a non-zero quantity when $b_{\gamma_{z}}$ is not averaged over a sufficient number of 2DZMs [Fig. 3(a1)]. In order to support this point, Fig. 6 displays the $\mathrm{BSC}_{\mathrm{CC}}$ computed by setting $b_{\gamma_{z}}(\Delta r)$ to zero outside the lag distance $0 \leq \Delta r \leq 2 \hat{a}$, as done previously by Luchies et $a .^{22}$ for sparse media. As expected, the $\mathrm{BSC}_{\mathrm{CC}}$ agree well with the expected $\mathrm{BSC}_{3 \mathrm{DFT}}$ at the lowest volume fraction of $0.6 \%$ when $b_{\gamma_{z}}(\Delta r \geq 2 \hat{a})=0$ [Fig. 6(a)]. However, the $b_{\gamma_{z}}$ cannot be set to zero for $\Delta r \geq 2 \hat{a}$ when considering higher volume fractions because it would create biases in $\mathrm{BSC}_{\mathrm{CC}}$ estimates as observed in Figs. 6(b) and 6(c).

The advantage of the 2DZM Fourier transform-based approach is its ability to capture information on 3D scatterer properties with a small number of 2DZM slices as demonstrated by satisfactory estimates of scatterer size distribution (Fig. 5). Moreover, this approach uses the fast Fourier transform algorithm, which is twice as fast to compute the $\mathrm{BSC}_{2 \mathrm{DFT}}$ than to compute the $\mathrm{BSC}_{\mathrm{CC}}$. However, the 2DZM Fourier transform-based approach does not allow estimating

TABLE I. QUS parameters estimated from the 2DZM correlation coefficient-based approach (denoted CC) and the 2DZM Fourier transform-based approach (denoted 2DFT). Columns 1 and 2 correspond to the simulated 3D media consisting of randomly oriented monodisperse prolate ellipsoids. Columns 3 and 4 correspond to the histological slices of HT29 cell pellets.

\begin{tabular}{|c|c|c|c|c|c|c|c|c|c|}
\hline \multirow{3}{*}{$\begin{array}{l}\text { QUS parameters } \\
a^{*} \text { in } \mu \mathrm{m}\end{array}$} & \multirow{3}{*}{$\frac{\text { Approach }}{\mathrm{CC}}$} & \multicolumn{2}{|c|}{1} & \multicolumn{2}{|c|}{2} & \multicolumn{2}{|c|}{3} & \multicolumn{2}{|c|}{4} \\
\hline & & \multicolumn{2}{|c|}{ Ellipsoids $\nu=1.5$} & \multicolumn{2}{|c|}{ Ellipsoids $\nu=2.0$} & \multicolumn{2}{|c|}{ Cell pellet 1} & \multicolumn{2}{|c|}{ Cell pellet 2} \\
\hline & & $4.2 \pm 0.04$ & $(4.5)$ & $3.7 \pm 0.02$ & $(4.5)$ & $4.5 \pm 0.34$ & $(4.6)$ & $4.8 \pm 1.05$ & $(4.5)$ \\
\hline & $2 \mathrm{DFT}$ & $4.3 \pm 0.05$ & $(4.5)$ & $3.8 \pm 0.01$ & $(4.5)$ & $4.6 \pm 0.06$ & $(4.6)$ & $4.5 \pm 0.11$ & $(4.5)$ \\
\hline \multirow[t]{2}{*}{$\sigma^{*}$ in $\mu \mathrm{m}$} & $\mathrm{CC}$ & $0.46 \pm 0.01$ & $(0)$ & $0.43 \pm 0.03$ & $(0)$ & $0.50 \pm 0.05$ & $(0.56)$ & $0.40 \pm 0.12$ & $(0.35)$ \\
\hline & 2DFT & $0.47 \pm 0.01$ & $(0)$ & $0.38 \pm 0.04$ & $(0)$ & $0.56 \pm 0.02$ & $(0.56)$ & $0.50 \pm 0.10$ & $(0.35)$ \\
\hline \multirow[t]{2}{*}{$\phi^{*}$ in $\%$} & $\mathrm{CC}$ & $29 \pm 0.5$ & $(30)$ & $23 \pm 1.1$ & $(30)$ & $37 \pm 10$ & (25) & $30 \pm 24$ & $(25)$ \\
\hline & 2DFT & $32 \pm 1.2$ & $(30)$ & $25 \pm 0.6$ & (30) & $32 \pm 1$ & $(25)$ & $32 \pm 0$ & $(25)$ \\
\hline \multirow[t]{2}{*}{$\gamma_{z}^{*}$} & $\mathrm{CC}$ & $0.11 \pm 0.00$ & $(0.11)$ & $0.12 \pm 0.00$ & $(0.11)$ & $0.07 \pm 0.01$ & $(0.11)$ & $0.08 \pm 0.05$ & $(0.11)$ \\
\hline & $2 \mathrm{DFT}$ & $0.11 \pm 0.00$ & $(0.11)$ & $0.12 \pm 0.00$ & $(0.11)$ & $0.10 \pm 0.00$ & $(0.11)$ & $0.10 \pm 0.00$ & $(0.11)$ \\
\hline
\end{tabular}



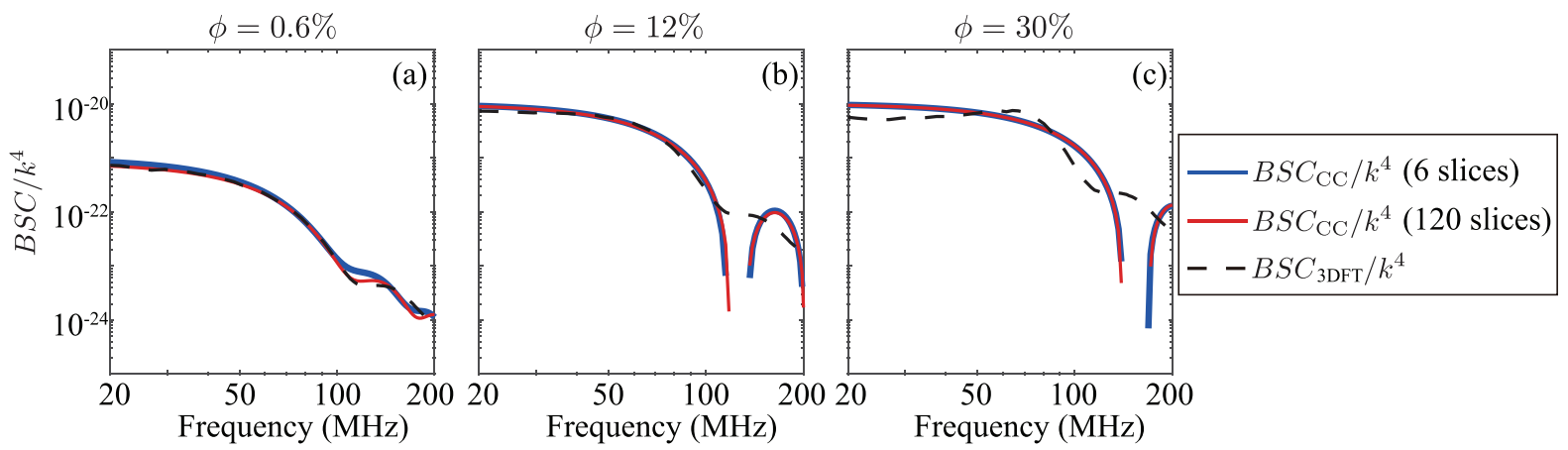

FIG. 6. (Color online) Same as Fig. 3(b), but the $\mathrm{BSC}_{\mathrm{CC}}$ is computed by setting $b_{\gamma_{z}}(\Delta r)$ to zero outside the lag distance $0 \leq \Delta r \leq 2 \hat{a}$.

the BSC in 3D space; it only allows estimating the BSC in $2 \mathrm{D}$ space. As a consequence, the fitting procedure to estimate the 3D scatterer properties uses the polydisperse SFM in 2D space. It is interesting to observe in Fig. 4(b) that the 2D BSC derived from the 2DZM and the 3D BSC derived from the 3DZM have the same behaviors: the maxima of $\mathrm{BSC}_{2 \mathrm{DFT}}$ and $\mathrm{BSC}_{3 \mathrm{DFT}}$ occur at the same frequencies and the amplitudes of $\mathrm{BSC}_{2 \mathrm{DFT}}$ and $\mathrm{BSC}_{3 \mathrm{DFT}}$ follow the same trend. The good agreement in maxima positions between $\mathrm{BSC}_{2 \mathrm{DFT}}$ and $\mathrm{BSC}_{3 \mathrm{DFT}}$ allows for the satisfactory estimation of the average sizes of 3D scatterers using the 2DZM Fourier transform-based approach.

The major result of this study is the validation of both 2DZM approaches to estimate the BSC (in 3D or 2D space) for concentrated discrete scatterers with uniform background. Further comparison between 2DZM and 3DZM should be conducted to confirm the added value of 2DZM when more complex 3D structures (such as blood microvessels and/or collagen fibers) are involved.

\section{B. QUS parameters from the 2DZM slices of simulated polydisperse spheres}

The present study incorporates the polydisperse SFM to estimate QUS parameters when using 2DZMs. Both 2DZM approaches using the polydisperse SFM (i.e., 2D Fourier transform using 6 2DZM slices and correlation coefficient using 120 2DZM slices) provide satisfactory QUS estimates for the simulated 3D media, consisting of polydisperse spheres at various volume fractions (Fig. 5).

In the case of dense media, the presence of tightly packed scatterers gives rise to coherent scattering [which is modeled by a structure factor $S(k, a, \phi)$ ], which strongly modifies the frequency dependence of the BSC. In addition, to permit an accurate estimation of the scatterer radius, this change in the BSC can be appropriately exploited to simultaneously estimate the volume fraction and the impedance contrast as shown previously in ultrasonic experiments on concentrated cell pellet biophantoms. ${ }^{16}$ Nevertheless, when the medium is sparse, the structure factor is equal to one at all frequencies and, therefore, the SFM inverse problem becomes ill-posed and unable to separate the distinct contribution of the volume fraction and impedance contrast and, consequently, QUS parameters are incorrectly estimated.
That is why the estimation of the impedance contrast is more accurate for dense media $(\phi \geq 6 \%)$ as shown in Fig. 5(d).

It is interesting to observe that the inversion procedure based on the polydisperse SFM yielded satisfactory estimates of the scatterer radii standard deviation $\sigma^{*}$ [see Fig. 5(b)]. This accurate estimation was feasible because of the use of a wide $20-200 \mathrm{MHz}$ frequency bandwidth in the inversion procedure, allowing the inclusion of the extrema of the BSC. This finding demonstrates the strength and value of the polydisperse SFM model approach with 2DZMs because of the large frequency bandwidth available.

The QUS parameters for the 3D simulated media consisting of polydisperse spheres were also estimated by fitting the BSC with the polydisperse sparse model. More specifically, the $\mathrm{BSC}_{\mathrm{CC}}$ was fitted with the polydisperse FFSM in $3 \mathrm{D}$ space, and the $\mathrm{BSC}_{2 \mathrm{DFT}}$ was fitted with the polydisperse fluid disc model in 2D space given by Eq. (A1) with $S(k)=1$. Results are presented in the supplemental figure. ${ }^{29}$ The scatterer sizes are largely underestimated for the dense media with actual volume fractions $\phi \geq 24 \%$ as expected. Indeed, the polydisperse sparse model neglects the coherent scattering and is incapable of accurately modeling scattering from dense media.

\section{QUS parameters from the 2DZM slices of more complex media (simulated ellipsoids and histology)}

When using the 2DZMs based on the simulated prolate ellipsoids (first two columns of Table I), the estimated scatterer radius agrees well with the expected effective radius when the actual axial ratio $\nu=1.5$ but not with the largest axial ratio $\nu=2.0$. In this last case $\nu=2.0$, the scatterer radius and surface fraction estimated by $2 \mathrm{DZM}$ approaches are underestimated. For frequencies less than $70 \mathrm{MHz}$, a decrease in $a$ has the effect of decreasing the BSC amplitude, and a decrease in $\phi$ has the effect of increasing the BSC amplitude for $\phi \geq 25 \%$ (dense media). That is why the underestimation of the radius is concomitant with that of the surface fraction. It is also interesting to observe that a polydispersity in size was found (i.e., $\sigma^{*} \geq 0.62 \mu \mathrm{m}$ ), whereas the actual scattering medium was monodisperse. A physical interpretation of this observed polydispersity is that the structure factor of a collection of randomly oriented 
monodisperse ellipsoids can be approximated by the structure factor of polydisperse spheres, as shown by Hansen. ${ }^{30}$

When using the 2DZM Fourier transform-based approach based on the histology photomicrographs of HT29 cell pellets, excellent agreement was obtained between estimated and actual nuclei sizes (relative error less than 5\%). The 2DZM correlation coefficient-based approach does not allow us to correctly estimate the nuclei size. This bad estimation and the large standard deviation obtained could be explained by the small number of 2DZMs used (i.e., six 2DZM slices) as suggested by the results obtained from 3D simulated media. Indeed, bias in scatterer size estimates can be obtained with the 2DZM correlation coefficient-based approach when the number of 2DZM slices is not sufficient [for example, see the scatterer sizes estimated for actual $\phi=24 \%$ in Fig. 5(a)].

The simulated 2DZMs based on the histology photomicrographs were performed to assess the 2DZM approaches on a more complex configuration but still close to dense collections of discrete scatterers. The good agreement between estimated and actual sizes obtained with ellipsoids $\nu=1.5$ or HT29 nuclei implies that QUS methods based on the SFM are flexible with respect to the shape of the scatterers. The model can still work reasonably well even if the scatterers are nonspherical or non-isotropic individually. However, it is important to note that a medium filled with randomly oriented ellipsoids is actually isotropic even though each scatterer is not, an assumption that may be violated in tissues where nonspherical cells may have a preferred orientation.

\section{Significance of 2DZM approaches for clinical and preclinical studies}

Another important axis of research is the use of experimentally obtained 2DZMs from acoustic microscopy. ${ }^{31-37}$ The 2DZMs obtained from acoustic microscopy systems operating at very high frequencies (i.e., > $250 \mathrm{MHz}$ ) provide realistic data to evaluate the $2 \mathrm{DZM}$ approaches in real tissues or even in cell pellet biophantoms but remain time consuming. In addition, the spatial resolution of the 2DZMs is approximately the wavelength at the center frequency of the transducer used to collect the data, potentially limiting the bandwidth used for model inversion. Nevertheless, acoustic microscopy studies are ongoing and could provide another insight in the methods proposed herein. Acoustic microscopy can provide the acoustic properties of tissues at different stages of pathology. ${ }^{33,34,37}$ Novel form factors derived from the 2DZMs could prove more accurate than previous standard form factors typically used for QUS studies. This ultimately would help with translation of QUS methods for in vivo studies where sensitivity and specificity to a particular disease could be improved by the use of better scattering models.

\section{CONCLUSION}

Two 2DZM approaches were compared to estimate 3D scatterer properties from 2DZMs: one based on the correlation coefficient and the other based on the 2D Fourier transform of 2DZMs. Both 2DZM approaches use the polydisperse SFM to estimate QUS parameters. Results demonstrate that both 2DZM approaches give similar 3D scatterer properties. The 2DZM correlation coefficient-based approach requires a large number of 2DZM slices, whereas the 2DZM Fourier transform-based approach is able to estimate 3D scatterer properties with a small number (i.e., six) of 2DZM slices. The critical configuration of simulated media for which the polydisperse SFM cannot accurately estimate the scatterer size is that of randomly oriented prolate ellipsoids with an axial ratio of 2 . In that case, further work in understanding the scattering from randomly oriented ellipsoids is required to better interpret the QUS parameters estimated with the polydisperse SFM.

The results demonstrate that the 2DZM Fourier transform-based approach is a valuable alternative to 3DZM for studying isotropic concentrated scattering media with a small number of 2DZM slices. The method based on the 2DZM Fourier transform-based approach could be highly relevant to experimental and (pre-) clinical studies because accurate 2DZMs can be directly obtained using state-of-theart acoustic microscopy systems and algorithms. ${ }^{31-37}$ Therefore, the proposed 2DZM approach could pave the way toward an easier and more complete understanding of ultrasound scattering in soft tissues, ultimately allowing a faster adoption of more reliable QUS methods for a wide variety of preclinical and clinical studies.

\section{ACKNOWLEDGMENT}

The authors thank Dr. François Destrempes for providing the analytical expression of the 2D structure factor for hard discs [based on the article by Guo and Riebel (Ref. 38)] and Mr. Lenin Chinchilla from Aix-Marseille University, Laboratory of Mechanics and Acoustics, for providing the spatial distribution of ellipsoids. This work was supported the Tateishi foundation, JSPS Core-to-Core Program, KAKENHI Grant Nos. 15H03030, 17H05280, and 19K20666, the Chiba University Global Program Research "Multi-modal medical engineering," and the A*MIDEX project (Grant No. ANR-11-IDEX-0001-02) funded by the "Investissements d'Avenir" French Government program.

\section{APPENDIX: 2D ULTRASONIC BACKSCATTERING THEORY}

This appendix presents the polydisperse SFM for an ensemble of 2D discs randomly distributed in 2D space with the goal of modeling ultrasound scattering from a perfectly thin tissue cross section. The discs represent the scatterers, which are assumed to be fluid and nonviscous media with an acoustic impedance $z_{1}$ in a surrounding background medium consisting of a (nonviscous) fluid with acoustic impedance $z_{0}$. (Note that such a $2 \mathrm{D}$ medium could also be thought of as a 3D medium, having no variation in the third dimension, 
i.e., the scatterers are infinite cylinders. This will be used below to derive the backscattering cross section of a single disc.)

For considering the polydispersity in terms of scatterer size, the scattering from a polydisperse system was approximated using the local monodisperse approximation. The local monodisperse approximation assumes that a scatterer of a certain size is always surrounded by scatterers of the same size. Under this hypothesis, the polydisperse medium is approximated by many monodisperse subsystems. The BSC for a mixture of discs differing only in size is calculated as the sum of the scatterings from the monodisperse subsystems weighted by the disc radius PDF $p(r)$, and is expressed as ${ }^{15}$

$$
\operatorname{BSC}_{\mathrm{SFM}}^{\mathrm{polyd} 2 \mathrm{D}}(k)=m \int_{0}^{\infty} p(r) \sigma_{b}(k, r) S(k, r, \phi) d r,
$$

where $k$ is the wavenumber and $m$ is the number density related to the total surface fraction $\phi$ as $m=\phi /\left(\pi \int_{0}^{\infty} p(r)\right.$ $\left.r^{2} d r\right)$. The backscattering cross section $\sigma_{b}$ of a single disc was calculated using the fluid infinite cylinder expression in the 2D case [see Eqs. (12) and (13) in Ref. 39]

$$
\sigma_{b}(k, r)=\frac{k^{3} A_{s}^{2} \gamma_{z}^{2}}{4 \pi^{2}}\left(\frac{J_{1}(2 k a)}{k a}\right)^{2},
$$

where $J_{1}$ is the first-order Bessel function of the first kind, $A_{s}$ is the disc area, and $\gamma_{z}$ is the relative impedance difference between the discs and the fluid surrounding medium $\gamma_{z}=\left(z_{1}-z_{0}\right) / z_{0}$. The function $S(k, r, \phi)$ in Eq. (A1) is the monodisperse structure factor for an equivalent system, consisting only of discs of radius $r$ with a fixed total surface fraction $\phi$, and obtained from the article by Guo and Riebel. ${ }^{38}$

${ }^{1}$ F. L. Lizzi, M. Greenebaum, E. J. Feleppa, M. Elbaum, and D. J. Coleman, "Theoretical framework for spectrum analysis in ultrasonic tissue characterization," J. Acoust. Soc. Am. 73(4), 1366-1373 (1983).

${ }^{2}$ F. L. Lizzi, M. Ostromogilsky, E. J. Feleppa, M. C. Rorke, and M. M. Yaremko, "Relationship of ultrasonic spectral parameters to features of tissue microstructure," IEEE Trans. Ultrason. Ferroelectr. Freq. Control 34(3), 319-329 (1987).

${ }^{3}$ M. L. Oelze and J. William D. O'Brien, "Application of three scattering models to characterization of solid tumors in mice," Ultrason. Imag. 28(2), 83-96 (2006).

${ }^{4}$ A. Han, R. Abuhabsah, R. J. Miller, S. Sarwate, and W. D. O’Brien, “The measurement of ultrasound backscattering from cell pellet biophantoms and tumors ex vivo," J. Acoust. Soc. Am. 134(1), 686-693 (2013).

${ }^{5}$ E. Feleppa, F. Lizzi, D. Coleman, and M. Yaremko, "Diagnostic spectrum analysis in ophthalmology: A physical perspective," Ultrasound Med. Biol. 12(8), 623-631 (1986).

${ }^{6}$ E. J. Feleppa, T. Liu, A. Kalisz, M. C. Shao, N. Fleshner, V. Reuter, and W. R. Fair, "Ultrasonic spectral-parameter imaging of the prostate," Int. J. Imag. Syst. Technol. 8,11-25 (1997).

${ }^{7}$ M. L. Oelze, W. D. O'Brien, J. P. Blue, and J. F. Zachary, "Differentiation and characterization of rat mammary fibroadenomas and $4 \mathrm{t} 1$ mouse carcinomas using quantitative ultrasound imaging," IEEE Trans. Med. Imag. 23, 764-771 (2004).

${ }^{8}$ J. Mamou, A. Coron, M. L. Oelze, E. Saegusa-Beecroft, M. Hata, P. Lee, J. Machi, E. Yanagihara, P. Laugier, and E. J. Feleppa, "Three-dimensional high-frequency backscatter and envelope quantification of cancerous human lymph nodes," Ultrasound Med. Biol. 37(3), 345-357 (2011).
${ }^{9}$ S. C. Lin, E. Heba, T. Wolfson, B. Ang, A. Gamst, A. Han, J. W. Erdman, W. D. O'Brien, M. P. Andre, C. B. Sirlin, and R. Loomba, "Noninvasive diagnosis of nonalcoholic fatty liver disease and quantification of liver fat using a new quantitative ultrasound technique," Clin. Gastroenterol. Hepatol. 13(7), 1337-1345 (2015).

${ }^{10}$ R. J. Lavarello, W. R. Ridgway, S. S. Sarwate, and M. L. Oelze, "Characterization of thyroid cancer in mouse models using highfrequency quantitative ultrasound techniques," Ultrasound Med. Biol. 39(12), 2333-2341 (2013).

${ }^{11}$ D. Savéry and G. Cloutier, "A point process approach to assess the frequency dependence of ultrasound backscattering by aggregating red blood cells," J. Acoust. Soc. Am. 110(6), 3252-3262 (2001).

${ }^{12} \mathrm{~V}$. Twersky, "Low-frequency scattering by correlated distributions of randomly oriented particles," J. Acoust. Soc. Am. 81(5), 1609-1618 (1987).

${ }^{13}$ E. Franceschini and R. Guillermin, "Experimental assessment of four ultrasound scattering models for characterizing concentrated tissuemimicking phantoms," J. Acoust. Soc. Am. 132(6), 3735-3747 (2012).

${ }^{14}$ A. Han and W. D. O'Brien, "Structure function estimated from histological tissue sections," IEEE Trans. Ultrason. Ferroelectr. Freq. Control 63(9), 1296-1305 (2016).

${ }^{15}$ E. Franceschini, R. Guillermin, F. Tourniaire, S. Roffino, E. Lamy, and J.-F. Landrier, "Structure factor model for understanding the measured backscatter coefficients from concentrated cell pellet biophantoms," J. Acoust. Soc. Am. 135(6), 3620-3631 (2014).

${ }^{16}$ E. Franceschini, R. de Monchy, and J. Mamou, "Quantitative characterization of tissue microstructure in concentrated cell pellet biophantoms based on the structure factor model," IEEE Trans. Ultrason. Ferroelectr. Freq. Control 63(9), 1321-1334 (2016).

${ }^{17}$ P. Muleki-Seya, R. Guillermin, J. Guglielmi, J. Chen, T. Pourcher, E. Konofagou, and E. Franceschini, "High-frequency quantitative ultrasound spectroscopy of excised canine livers and mouse tumors using the structure factor model," IEEE Trans. Ultrason. Ferroelectr. Freq. Control 63(9), 1335-1350 (2016).

${ }^{18}$ E. Franceschini, J-M. Escoffre, A. Novell, L. Auboire, V. Mendes, Y. M. Benane, A. Bouakaz, and O. Basset, "Quantitative ultrasound in ex vivo fibrotic rabbit livers," Ultrasound Med. Biol. 45(7), 1777-1786 (2019).

${ }^{19}$ J. Mamou, M. L. Oelze, W. D. O'Brien, and J. F. Zachary, "Identifying ultrasonic scattering sites from three-dimensional impedance maps," J. Acoust. Soc. Am. 117(1), 413-423 (2005).

${ }^{20}$ J. Mamou, M. L. Oelze, W. D. O’Brien, and J. F. Zachary, "Extended three-dimensional impedance map methods for identifying ultrasonic scattering sites," J. Acoust. Soc. Am. 123(2), 1195-1208 (2008).

${ }^{21}$ A. D. Pawlicki, A. J. Dapore, S. Sarwate, and W. D. O’Brien, "Threedimensional impedance map analysis of rabbit liver," J. Acoust. Soc. Am. 130(5), EL334-EL338 (2011).

${ }^{22}$ A. C. Luchies and M. L. Oelze, "Using two-dimensional impedance maps to study weak scattering in sparse random media," J. Acoust. Soc. Am. 139(4), 1557-1564 (2016).

${ }^{23}$ A. C. Luchies and M. L. Oelze, "Effects of the container on structure function with impedance map analysis of dense scattering media," J. Acoust. Soc. Am. 143(4), 2172-2181 (2018).

${ }^{24}$ M. F. Insana and D. G. Brown, "Acoustic scattering theory applied to soft biological tissues," in Ultrasonic Scattering in Biological Tissues, edited by K. K. Shung and G. A. Thieme (CRC Press, Boca Raton, FL, 1993), Chap. 4, pp. 76-124.

${ }^{25}$ Note that the denominator in Eq. (2) is equal to $2 \pi$ [and not equal to $8 \pi$ as written in Eq. (65) in Ref. 24] because we consider the relative contrast in acoustic impedance (and not the relative contrasts in compressibility and density).

${ }^{26} \mathrm{~K}$. Kanatani and O. Isikawa, "A study of numerical schemes for the stereological estimation of particle size distribution," J. Soc. Powder Technol. Jpn. 21(3), 143-152 (1984)

${ }^{27}$ R. Saha and G. Cloutier, "Monte Carlo study on ultrasound backscattering by three-dimensional distributions of red blood cells," Phys. Rev. E 78, 061919 (2008).

${ }^{28}$ A. Dapore, L. A. Wirtzfeld, M. R. King, S. Sarwate, J. Harter, M. L. Oelze, T. J. Hall, M. N. Do, and W. D. O'Brien, "Analysis of human fibroadenoma using three-dimensional impedance maps," IEEE Trans. Med. Imag. 30(6), 1206-1213 (2011).

${ }^{29}$ See supplementary material at http://doi.org/10.1121/10.0001972 for figures showing the correlation coefficients $b_{\gamma_{\text {s }}}$ obtained from 1,6 , or 120 2DZM slices and a figure showing the QUS parameters estimated by the 


\section{JASA}

2DZM correlation coefficient-based approach or the 2DZM Fourier transform-based approach when using the theoretical polydisperse sparse model.

${ }^{30} \mathrm{~S}$. Hansen, "Approximation of the structure factor for nonspherical hard bodies using polydisperse spheres," J. Appl. Crystallogr. 46(4), 1008-1016 (2013).

${ }^{31}$ D. Rohrbach, A. Jakob, H. O. Lloyd, S. H. Tretbar, R. H. Silverman, and J. Mamou, "A novel quantitative 500-MHz acoustic microscopy system for ophthalmologic tissues,” IEEE Trans. Biomed. Eng. 64(3), 715-724 (2017).

${ }^{32}$ D. Rohrbach, K. Ito, H. O. Lloyd, R. H. Silverman, K. Yoshida, T. Yamaguchi, and J. Mamou, "Material properties of human ocular tissue at 7- $\mu$ m resolution," Ultrason. Imag. 39(5), 313-325 (2017).

${ }^{33}$ S. Irie, K. Inoue, K. Yoshida, J. Mamou, K. Kobayashi, H. Maruyama, and T. Yamaguchi, "Speed of sound in diseased liver observed by scanning acoustic microscopy with $80 \mathrm{MHz}$ and $250 \mathrm{MHz}$," J. Acoust. Soc. Am. 139(1), 512-519 (2016).

${ }^{34}$ Q. V. Hoang, D. Rohrbach, S. A. McFadden, and J. Mamou, "Regional changes in the elastic properties of myopic Guinea pig sclera," Exp. Eye Res. 186, 107739 (2019).
${ }^{35}$ D. Rohrbach and J. Mamou, "Autoregressive signal processing applied to high-frequency acoustic microscopy of soft tissues," IEEE Trans. Ultrason. Ferroelectr. Freq. Control 65(11), 2054-2072 (2018).

${ }^{36}$ Y. Saijo, E. S. Filho, H. Sasaki, T. Yambe, M. Tanaka, N. Hozumi, K. Kobayashi, and N. Okada, "Ultrasonic tissue characterization of atherosclerosis by a speed-of-sound microscanning system," IEEE Trans. Ultrason. Ferroelectr. Freq. Control 54(8), 1571-1576 (2007).

${ }^{37}$ K. Miura, H. Nasu, and S. Yamamoto, "Scanning acoustic microscopy for characterization of neoplastic and inflammatory lesions of lymph nodes," Sci. Rep. 3, 1255 (2013).

${ }^{38} \mathrm{X}$. Guo and U. Riebel, "Theoretical direct correlation function for twodimensional fluids of monodisperse hard spheres," J. Chem. Phys. 125(14), 144504 (2006).

${ }^{39}$ E. Franceschini, B. Metzger, and G. Cloutier, "Forward problem study of an effective medium model for ultrasound blood characterization," IEEE Trans. Ultrason. Ferroelectr. Freq. Control 58(12), 2668-2679 (2011). 